\title{
New Patents on Topical Anesthetics
}

\author{
Carmen Cantisani ${ }^{1, *}$, Laura Macaluso ${ }^{1}$, Federica Frascani ${ }^{1}$, Giovanni Paolino ${ }^{1}$, Vito D’ Andrea ${ }^{2}$, Antonio G. \\ Richetta $^{1}$ and Stefano Calvieri ${ }^{1}$
}

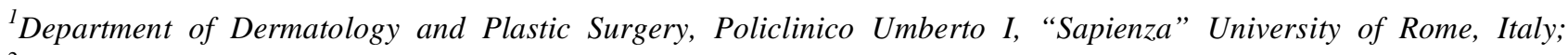
${ }^{2}$ Dipartimento di Scienze Chirurgiche, Policlinico Umberto I, "Sapienza” University of Rome, Italy,

Received: November 26, 2013; Accepted: May 14, 2014; Revised: May 14, 2014

\begin{abstract}
Anesthesia is defined as a total or partial loss of sensation and it may be general, local or topical, depending on the method of drug administration and area of the body affected. General anesthesia is a reversible state of unconsciousness produced by anesthetic agents, characterized by amnesia, muscle relaxation and loss of sensitivity to pain of the whole body. General anesthetic drugs can be classified into two main groups according to their predominant molecular pharmacological effects: volatile and intravenous agents. Local anesthesia produce a reversible loss of sensation in a portion of the body and it reversibly block impulse conduction along nerve axons and other excitable membrane. All local anesthetics (LA) are membrane stabilizing drugs; they reversibly decrease the rate of depolarization and repolarization of excitable membranes. They act mainly by inhibiting sodium influx through sodium-specific ion channels in the neuronal cell membrane, in particular the voltage-gated sodium channels. When the influx of sodium is interrupted, an action potential cannot arise and signal conduction is inhibited. The main local anesthetic (LA) agents for skin anesthesia are benzocaine (aminoester), prilocaine and lidocaine (aminoamides) which are commercially available as gels, ointments and creams (benzocaine and eutectic mixture of lidocaine and prilocaine) or as a bioadhesive (lidocaine) with different compositions (vehicles and excipients) for adults or pediatric use. Topical anesthetics decrease anxiety, pain and discomfort during cutaneous procedures and provide effective analgesia with rapid onset, prolonged duration and minimal side effects. This article outlines the different classes of topical anesthetics available and gives an overview of the mechanism of action, metabolism of each different class, of the possible complications that can occur because of their use and their possible treatment options and new patents.
\end{abstract}

Keywords: Analgesia, dermatologic procedures, muscle relaxation, new patents, pain, topical anesthetics,

\section{INTRODUCTION}

Anesthesia is defined as a loss of sensation and it may be general, local or topical, depending on the method of drug administration and area of the body affected [1]. The development of safe and effective local anesthetic agents has possibly been one of the most important advancements in medicine to occur in the last century.

\section{General Anesthetics}

General anesthesia is a reversible state of unconsciousness produced by anesthetic agents, characterized by amnesia, muscle relaxation and loss of sensitivity to pain of the whole body. General anesthetic drugs can be classified into two main groups according to their predominant molecular pharmacological effects: volatile and intravenous agents.

*Address correspondence to this author at the Department of OncoematologyDermatology and Plastic Surgery. Azienda Policlinico Umberto I, University "Sapienza", viale del Policlinico 155, 00167 Rome Italy; Alternative address: Via di Boccea n. 10 int 1600167 Rome, Italy; Tel: +39-3479385719; Fax: +39-06490243; E-mails: carmencantisanister@gmail.com;

carmen.cantisani@uniroma1.it
Volatile anesthetics were first introduced into clinical use in 1842 and can be divided into organic and inorganic agents. The commonly used volatile anesthetics in current clinical practice include isoflurane, halothane, desflurane and sevoflurane. They bind to many body proteins modifying their functions and producing an anesthetic action. Several protein targets have been identified for these agents such as ion channels, muscarinic acetylcholine receptors, opioid receptors, alpha2 adrenergic receptors, 5-hydroxytryptophan receptors and $N$-methyl-D-aspartate (NMDA) receptors [1]. Volatile agents provide all components of general anesthesia but they also have additional properties. Many animal studies have shown that these drugs are able to reduce inflammatory processes induced by various stimuli, including ischemia and inflammation inducing agents. Similar findings have been revealed in humans. Data of literature have reported, in fact, that sevoflurane $2 \%$ inhibits the function of inflammatory cells by decreasing the expression of TNFa and other cytokines such as IL-6 and IL-8 [2]. Nevertheless, current evidence shows dual effects of volatile anesthetics on inflammatory process: they can inhibit inflammation induced by several potentially harmful stimuli (e.g., surgery) and induce neu- 
roinflammation in the absence of stimuli. Little is known for the mechanisms of volatile anesthetics-induced neuroinflammation. Isoflurane has been shown to open the blood brain barrier. This can increase the permeation of intravascular substances to brain tissues. Furthermore, isoflurane seems to activate NF-kB, a transcription factor known to increase inflammatory cytokine production, causing neuroinflammation that then leads to cognitive impairment [3]. As regards their side effects, in literature are reported the following: increased intracranial pressure, hypotension, cardiac arrhythmias, airway irritability and depression of mucociliary system [1].

Intravenous anesthetics were introduced into clinical use for the first time in 1939 [1]; they can be divided into barbiturates and non-barbiturates agents. Barbiturates are a class of drugs that act on the $\mathrm{GABA}_{\mathrm{A}}$ receptor in the brain and spinal cord. The $\mathrm{GABA}_{\mathrm{A}}$ receptor is an inhibitory channel that decreases neuronal activity, and barbiturates enhance the inhibitory action of the $\mathrm{GABA}_{\mathrm{A}}$ receptor. Barbiturates have anesthetic, sedative, anxiolytic, anticonvulsant and hypnotic properties but do not have analgesic effects. The main representative agent of this class is thiopentone. As with nearly all anesthetic drugs, thiopentone causes cardiovascular and respiratory depression resulting in hypotension, apnea and airway obstruction. Side effects also include headache, agitated emergence, prolonged somnolence, and nausea [1]. Among the non-barbiturates anesthetics, the most commonly used are propofol and ketamine. Propofol has been proposed to have several mechanisms of action both through potentiation of $\mathrm{GABA}_{\mathrm{A}}$ receptor activity, thereby slowing the channel-closing time, and also acting as a sodium channel blocker [1]. Recent research has also suggested that the endocannabinoid system may contribute significantly to propofol's anesthetic action and to its unique properties [4]. Aside from low blood pressure related to vasodilation, and transient apnea following induction doses, one of propofol's most frequent side effects is pain on injection, especially in smaller veins. This pain arises from activation of the pain receptor, TRPA1, found on sensory nerves, and it can be mitigated by pretreatment with lidocaine [5]. Other rare side effects are dystonia and priapism. Ketamine is classified as an NMDA receptor antagonist, but it also interacts with muscarinic receptors, descending monoaminergic pain pathways and voltage-gated calcium channels [1]. In literature short term and long term side effects are reported. The first ones occur immediately after the operation and they are transitory: delirium, dizziness, diplopia, blurred vision, nystagmus, tachycardia, hypersalivation, nausea and vomiting, erythema, aphasia, psychomotor retardation, nightmares, hallucinations and tonic-clonic movements. Long term side effects arise a few months after the anesthesia and they mainly comprise neurological effects (cognitive impairments, depression memory problems) and urinary tract effects (incontinence, decreased bladder compliance, detrusor overactivity, painful haematuria, bilateral hydronephrosis and renal papillary necrosis) [6]. Inhalational and intravenous anesthetics may both cause and mitigate existing significant neuropathology and they may cause neuroapoptosis, caspase activation, neurodegeneration, $\beta$-amyloid protein (A $\beta$ ) accumulation and oligomerization, and ultimately, deficits in neurocognition [7].

\section{Local Anesthetics}

Local anesthesia produces a reversible loss of sensation in a portion of the body and it reversibly blocks impulse conduction along nerve axons and other excitable membranes. Local anesthetics (LA) interrupt neural conduction by inhibiting the influx of sodium ions through channels or ionophores within neuronal membranes. When the influx of sodium is stopped, an action potential cannot arise and signal conduction is inhibited. The use of LA dates back to the second half of the nineteenth century. These drugs vary in their pharmacological properties and they are used in various techniques of local anesthesia such as: topical anesthesia (surface), infiltration, plexus block, epidural (extradural) block and spinal anesthesia (subarachnoid block). LA can be classified as ester- or amide-derived local anesthetics. Their basic structure consists of an aromatic lipophilic ring and an amine hydrophilic group connected by an intermediate hydrocarbon chain. Ester and amide anesthetics differ in their chemical stability and metabolism. The amino esters are relatively unstable in solution and are easily hydrolyzed in the body by plasma cholinesterase and other nonspecific esterases. One metabolite formed by this ester hydrolysis reaction is para-aminobenzoic acid (PABA), which is capable of causing allergic reactions in a small percentage of patients. In contrast, the amino amides are much more stable and undergo enzymatic degradation in the liver. Because they are not metabolized to PABA, allergic reactions to the amidetype local anesthetics are extremely rare [1]. The amino amides include lidocaine, mepivacaine, prilocaine, bupivacaine, etidocaine, ropivacaine; the amino esters comprehend cocaine, procaine, clorprocaine, tetracaine and benzocaine. Systemic toxicity includes side effects on the central nervous system and the heart. As local anesthetics are absorbed from the injection site, their concentration in the bloodstream rises and the peripheral nervous system (PNS) and central nervous system (CNS) are depressed in a dose-dependent manner. Although all local anesthetics carry comparable risk for CNS toxicity, it should be noted that bupivacaine exhibits greater potential for direct cardiac toxicity than other agents [8]. Even levobupivacaine and ropivacaine (single-enantiomer derivatives) developed to ameliorate cardiovascular side effects, still harbor the potential to disrupt cardiac function [9]. The systemic toxicity of prilocaine is comparatively low, however its metabolite, $O$-toluidine, is known to cause methemoglobinemia. As methemoglobinemia reduces the amount of hemoglobin that is available for oxygen transport, this side effect is potentially life-threatening. Therefore, dose limits for prilocaine should be strictly observed [10]. Local anesthetics may also cause allergic reactions. In literature are reported either a syncopal episode associated with the injection, or cardiac palpitations attributed to epinephrine contained either in the solution or released endogenously. Allergic reactions following local anesthetic injections are more likely attributable to preservatives (methylparaben) or antioxidants (sulfites) contained in the solution [11]. Local anes- 
thetics could also induce histological damage with lesions ranging from local to extreme in skeletal muscle. Metabolic alterations were also described involving sarcoplasmic reticulum and calcium dysregulation, alteration of mitochondrial physiology and of oxidative phosphorylation with associated overproduction of harmful reactive oxygen species, causing apoptosis or necrosis [12].

\section{Topical Anesthetics}

Topical anesthesia is one of the most used strategies to minimize anxiety, pain and discomfort at the site of the needle insertion during dermatological surgical procedures. The main topical anesthetic agents for skin anesthesia are benzocaine (aminoester), prilocaine and lidocaine (aminoamides) which are commercially available as gels, ointments and creams (benzocaine and eutectic mixture of lidocaine and prilocaine) or as a bioadhesive (lidocaine) with different compositions (vehicles and excipients) for adults or pediatric use. The purpose of such topical formulations is to promote the permeation at site of application and a rapid onset of action, enhancing the topical anesthetic effect and reducing the pain associated with dermatological procedures. The first topical anesthetic mixture reported was composed $0.5 \%$ tetracaine, $0.05 \%$ epinephrine and $11.8 \%$ cocaine. The high local and systemic toxicity limited its use, and a mixture of $4 \%$ lidocaine, $0.5 \%$ tetracaine and $0.1 \%$ epinephrine was proposed for replacing cocaine. In 1992, the eutectic mixture of $2.5 \%$ lidocaine and $2.5 \%$ of prilocaine known as EMLA was approved by the US FDA, being the main anesthetic formulations for skin anesthesia due to its efficacy and safety for a variety of procedures in dermatology. Topical anesthetics efficacy has been evaluated by different methods such as pinprick and tail flick tests in pre-clinical studies. At the same way, tactile spatial discrimination, puncture of intact skin and tape-stripping technique have been used for clinical efficacy evaluation. Topical formulations containing LA are usually applied on the skin for analgesia or for the management of acute and chronic superficial pain. Most of the formulations available are liquid or semisolids. The main drawback of these formulations is that they can be easily removed from the skin surface by rubbing of clothing or inadvertent touching, reducing the contact time with the skin and consequently modifying the efficacy. A relatively new alternative formulation is represented by patches that normally consist of an outer covering which support a preparation that contains the active substance intended to be applied to intact skin to obtain a local and systemic delivery. Unlike the traditional topical formulations, the patches guarantee the control of the applied dose, of the area of application, of the contact time and of the release kinetics. The first patch containing topical anesthetics, namely lidocaine, was approved by FDA in 1999 for the treatment of postherpetic neuralgia. The lidocaine patch $5 \%$ consists of a thick layer of adhesive hydrogel containing the active applied on a woven-non woven backing and covered by a polyethylene terephthalate release liner. Lidocaine patch is nowadays a first-line treatment of postherpetic neuralgia or other localized pain conditions and its efficacy was demonstrated by many studies. The development of topical anesthetic formulations and its widespread use has become an alternative to reduce pain and anxiety related to invasive procedures, such as venipuncture, pinprick testing, and split-thickness skin graft donation. Combined anesthetic agents have been widely used in the clinical practice for skin anesthesia. The combination between topical anesthetics agents and/or vasoconstrictors was the first strategy for skin anesthesia. Nowadays, in order to provide a fast onset and to prolong the duration of anesthesia, some of the different strategies purposed include liposomes, lipid nanoparticles, formulations of hydrogels and also highly water permeable patches have been reported in a variety of studies, pulled together here. Liposomes are microscopic, spherical, phospholipid-based carriers that deliver a greater concentration of local anesthetic to sensory nerves than other conventional formulations with lower lipophilicity [13]. However, despite the availability of different procedures and formulations, the development of an ideal topical anesthetic formulation for skin-delivery is still a challenge. The main limitation is the stratum corneum barrier due to its lipid phase composition and organization. Several techniques can improve dermal absorption of topical anesthetics. Removing the stratum corneum with preoperative procedures such as tape stripping, degreasing with acetone or laser ablation enhances dermal absorption. Occlusion and heat can also facilitate anesthetic penetration into the skin. Iontophoresis increases absorption of topical anesthesia by using an electric current to facilitate the passage of ionized local anesthetic into and across the skin barrier. Adding epinephrine to local anesthetic induces vasoconstriction, which slows the anesthetic's removal and raises the duration of its local tissue effect [14]. Toxic reactions to topically applied anesthetics correlate with their peak blood levels. Peak blood levels are achieved more quickly with topical anesthetics applied to mucous membranes and large areas of skin. If a large area of skin is involved, treatments should be divided into smaller anatomic portions so that appropriately sized segments of skin are anesthetized and treated during each session. Improper applications of topical anesthetic formulations such as benzocaine, lidocaine, and tetracaine may cause serious complications, including cardiotoxicity and central nervous system toxicity [15].

\section{SKIN ANESTHETICS}

\section{Eutectic Mixture of Local Anesthetics}

The topical anesthetic agent widely used is a eutectic mixture of local anesthetics (EMLA; Astra Pharmaceuticals, Westborough, MA). EMLA is an oil-in-water emulsion of $2.5 \%$, lidocaine and $2.5 \%$ prilocaine wich contains polyoxyethylene fatty acid esters functioning as emulsifier, which enhance the skin penetration by increasing the effective concentration of anesthetics in the oil droplet to $80 \%$. EMLA is often applied to the skin under an occlusive dressing, which facilitates absorption; it has a recommended 1hour minimal application time on intact skin, although the onset of cutaneous analgesia depends on the anatomic site. For example, EMLA is ineffective on palmar and plantar areas, while, for the mucous membranes (genitalia, lips), effective anesthesia can usually be obtained within 5 to 15 
minutes without occlusion. The depth of the analgesia is highly correlated with the duration of application. For particular painful procedures, such as skin graft harvesting, the recommended time is at least 2 hours. Dermal analgesia reaches maximum at 2 to 3 hours after application and persists 1 to 2 hours after removal [16]. EMLA has a biphasic action on cutaneous blood vessels, with a vasoconstrictive effect that is maximal after 1.5 hours, followed by vasodilatation 2 to 3 hours after application. EMLA works by abolishing pain and decreasing the itch sensation and it is able to relieve the pain associated with venopuncture and venous cannulation, biopsies, curettage of molluscum contagiosum, split-thickness skin grafts, and debridement of chronic leg ulcers, chemical peels, epilation, and vascular laser treatments. The maximum recommended dose and application area are based on patient's age, body weight, intact skin, and normal renal and hepatic function. The concentrations associated with systemic toxicity are in the region of $6 \mu \mathrm{g} / \mathrm{mL}$. The major concern regarding the use of EMLA relates to the potential risk of methemoglobinemia. It seems that the risk of developing this side effect is mainly due to the prilocaine portion of EMLA because of its ability to oxidize iron in red blood cells from the ferrous to the ferric state, compromising hemoglobin transport of oxygen. When levels of methemoglobin are between $15 \%$ and $30 \%$, patients present with initial signes of cyanosis, including dyspnea, tachycardia, and headache. Methemoglobin levels greater than 50\% are associated with lethargy and coma [17]. Hypersensitivity reactions and systemic toxicity from EMLA are extremely rare. The use of EMLA is contraindicated in patients with a known history of sensitivity to amide-type local anesthetics. Because it is metabolized in the liver, patients with liver disease should consider it a relative contraindication. Patients with skin disease such as atopic dermatitis and psoriasis may have higher rates of absorption and side effects. EMLA is safe to administer on intact skin of full-term neonates, but care must be taken to limit the dose and area of application. Oral ingestion may lead to toxicity because of rapid systemic uptake via the mucous membrane. Minor application-site side effects include paleness, redness, and alteration in temperature sensations, edema, itching, and rash. Local reactions can be exacerbated by repeated applications but usually resolve after discontinuation. Eye contact with EMLA should be avoided because it contains sodium hydroxide that may cause alkaline chemical injury [18].

\section{ELA-Max}

ELA-Max (Ferndale Laboratories, Ferndale, Mich) is a topical anesthetic cream with $4 \%$ lidocaine at $\mathrm{pH}$ 7.4. Each gram of ELA-Max cream contains lidocaine $(40 \mathrm{mg}$ ), vitamin $\mathrm{E}$ acetate, propylene glycol, benzyl alcohol, lecithin, cholesterol, carbomer 940, triethanolamine, polysorbate 80, and purified water. It is used for the temporary relief of pain associated with minor cuts and abrasions of the skin, minor burns, and skin irritation. It is not recommended for mucous membrane use because limited studies have shown greater absorption of lidocaine than through intact skin.
The onset, depth, and duration of cutaneous analgesia depend primarily upon the duration of application, and the amount of lidocaine systemically absorbed is directly related to these variables.

The incidence of adverse reactions is limited. Topical adverse reactions consist of irritation and erythema, so eye contact with ELA-Max should be avoided. The low rate of systemic side effects is due to low peak blood levels. Toxic levels occur at more than $5 \mu \mathrm{g} / \mathrm{mL}$ for lidocaine and cause decreases in cardiac output, total peripheral resistance, and mean arterial pressure. Overdose symptoms may include also seizures, respiratory failure, and coma [19].

\section{Lidocaine}

Lidocaine, alone or in combination with another anesthetic, is the most widely used topical anesthetic. It belongs to the amide class of anesthetics, which has less risk of inducing an allergic reaction than ester anesthetics. Brand names for OTC topical lidocaine include Topicaine (ESBA Laboratories, Jupiter, FL), Lidoderm (Endo Pharmaceuticals, Chadds Ford, PA), and LMX (Ferndale Laboratories, Ferndale, MI). Topicaine is a $4 \%$ or $5 \%$ lidocaine gel, and Lidoderm is a $5 \%$ lidocaine adhesive patch. LMX is a $4 \%$ or 5\% lidocaine liposomal delivery cream that has several lipid bilayers, which facilitate dermal penetration of anesthetic and protect the drug from being rapidly metabolized, allowing LMX to achieve the same analgesia as EMLA in a shorter period of time without requiring occlusion [20].

Lidocaine is excreted through breast milk, so caution should be practiced when being administered to a nursing mother. Topical adverse effects can occur and they mainly appear as erythema, irritation and itching. Care must be exercised with use of topical lidocaine around the eye because accidental eye exposure will result in severe corneal irritation.

Prolonged applications, use of inappropriately high concentrations, and distribution to large surface areas increase the risk of cardiotoxicity and central nervous system toxicity. The initial symptoms of lidocaine-induced toxicity (for blood levels of $1-5 \mu \mathrm{g} / \mathrm{mL}$ ) include lightheadedness, circumoral numbness, diplopia, and tinnitus. When levels of lidocaine are between 20 and $25 \mu \mathrm{g} / \mathrm{mL}$, patients present with respiratory depression or coma. Many adverse reactions also appear to be related to the inclusion of epinephrine within the anesthetic mixture [21]. Several preparations of topical lidocaine are available for anesthesia of oral mucous membranes: $2 \%$ viscous solution, $5 \%$ liquid and 5\% ointment (Xylocaine, Astra Inc, Westborough, Mass). These topical agents are recommended for the prevention and treatment of oral mucosa pain, especially in children. The main indications are symptomatic treatment of minor injuries (such as trauma or aphtous ulcers) of oropharynx membranes or prevention of iatrogenic pain produced during painful dental procedures such as anesthetic injection or clamp placement [22]. 


\section{S-Caine Peel and S-Caine}

Patch An S-Caine peel, marketed as Pliaglis (Galderma Laboratories, Fort Worth, TX), was a eutectic mixture of 7\% lidocaine and $7 \%$ tetracaine in a cream base. It was discontinued in September 2008 because of an inability to obtain consistent product viscosity.

Plans for its re-release are unclear. The S-Caine Patch (Zars, Inc., Salt Lake City, UT) contains a small amount of topical anesthetic under a patented heating element that raises the temperature to $40^{\circ} \mathrm{C}$ for longer than 14 hours and enhances delivery of the anesthetic. This product is marketed as Synera and contains a eutectic mixture of $70 \mathrm{mg}$ of lidocaine and 70mg of tetracaine. When the patch is applied to the skin, it increases the skin temperature approximately $5^{\circ} \mathrm{C}$, but the maximum skin temperature produced by the patch at the site of application does not exceed $40^{\circ} \mathrm{C}$ [23]. The product manufacturer states that the patch should be placed on intact skin 30 minutes before superficial dermatologic procedures such as a shave biopsy. Erythema, blanching, and edema are the most commonly observed local reactions.

\section{MUCOUS MEMBRANE AND OPHTHALMIC ANES- THETICS}

Among topical anesthetics, it is worth mentioning the mucous membrane and the ophthalmic anesthetics. Mucous membrane anesthetics include Benzocaine and Cocaine. The first one exists in liquid, gel and spray formulations for oral anesthesia. It is used for treatment of minor mouth or gum wounds and inflammation. It is an ester local anesthetic; hence there is a small risk of the development of contact allergy. Adverse systemic reactions are uncommon but it can potentially induce methemoglobinemia [24]. Cocaine is the active alkaloid derived from the leaves of the Erythroxylon coca plant of South America. It lessens the sensibility to pain and diminishes the acuity of taste and smell. Topical cocaine produces anesthesia by preventing nerve impulse conduction and causes intense vasoconstriction by blocking the presynaptic reuptake of norepinephrine. Maximum local anesthesia occurs within 5 minutes and persists for up 60 minutes. Cocaine is highly effective on mucous membranes, but it is no well absorbed through intact skin; it is commonly used for topical anesthesia of the oral and nasal cavities and larynx [25].

Allergic reactions are uncommon. More frequent is the necrosis of nasal septum in patients who abuse cocaine. As regard systemic effects, cocaine may induce euphoria and increased alertness. Its sympathomimetic effects include hypertension, hyperthermia and tachycardia. Cocaine overdose (200 mg) can lead to convulsion, CNS depression respiratory failure and death.

\section{Ophthalmic Anesthetics Include Proparacaine and Tet- racaine}

Proparacaine's dermatologic indications are for removal of superficial foreign bodies from the cornea and short op- erative procedures involving the eyelid. Proparacaine is a rapidly acting acid ester anesthetic. The usual dose is 1 or 2 drops of $0.5 \%$ solution before the procedure; the onset of anesthesia usually begins in 30 seconds and persists for about 15 minutes. At usual dosages, proparacaine produces minimal local side effects. However, with prolonged use, it may retard wound healing and cause corneal epithelial erosions [26]. In literature are also described few cases of allergic contact dermatitis [27]. Systemic effects from procaine are rare; however toxicity may occurs with the stimulation of CNS, followed by CNS and cardiovascular depression. Tetracaine is employed in a $0.5 \%$ solution for topical use. It is a para-aminobenzoic acid ester whose mechanism of action is similar to that of propacain. The main side effect of tetracaine is the burning sensation encountered during the 30 seconds after instillation into the conjunctival sac. Local toxicity to tetracaine may occur after repeated use. Dangerous overdosage (higher than $1.5 \mathrm{mg} / \mathrm{Kg}$ of the body weight) can produce side effects similar to those of proparacaine [28].

\section{CURRENT \& FUTURE DEVELOPMENTS}

Thanks to the more widely use of these agents' new patents are developing in recent years; we will review them. An interesting discovering was treating neutrophil-associated pulmonary disease administering a topical anesthetic e.g. lidocaine, locally to the respiratory tract. A therapeutic method is provided to treat neutrophil-associated pulmonary diseases, such as chronic obstructive pulmonary disease, by locally administering an effective amount of a topical anesthetic, such as lidocaine, to counteract the symptoms of the disease [29].

Another invention useful for ameliorating neurogenic tremor e.g., kinetic, postural, task specific or resting, in mammals, comprises topically administering anesthetic [30].

A method for providing applicator of e.g., topical anesthetic for dental patient, involves attaching absorbent material at end of cap, such that medicinal substance is applied to material, and end of cap with material is applied to body [31].

Another new invention included transdermal analgesic applicator configured to provide a visual signal subsequent sufficient absorption of an analgesic compound ensuing the transdermal analgesic applicator being applied to the skin of a patient. The transdermal analgesic applicator includes a first layer that is impermeable and has integrally formed therewith on at least a portion thereof a plurality of microcapsules containing a thermochromic dye mixture. The first layer having the portion of microcapsules presents a first color at ambient room temperature and a second color at temperature greater than the first temperature as a result of being placed on the skin of a patient. A second layer containing an analgesic compound is secured to the first layer. The first layer changes from the first color to the second color subsequent the first layer changing from the first temperature to the second temperature, which the rate of temperature 
change is approximately equivalent to the absorption rate of the analgesic compound [32].

The various embodiments of the present inventions provide stabilization devices and methods for use of the stabilization devices with minimally invasive gynecological procedures such as methods of preventing pregnancy by inserting intrafallopian contraceptive devices into the fallopian tubes full of topical anesthetics. A minimally invasive method of sterilization, the method comprising: coupling a handle of an elongated catheter to an endoscope, the endoscope having a handle and an elongated portion designed for insertion into a patient, and the elongated catheter having an outer portion, an inner portion within the outer portion, and a corewire within the inner portion, wherein the outer portion and inner portion are slideable relative each other; inserting the elongated catheter and the endoscope into a uterus of the patient; inserting a sterilization device coupled to a distal portion of the elongated catheter into a fallopian tube of the patient, the sterilization device having an outer expandable portion; moving a release mechanism on the handle from a first incremented position having a first labeled operation to a second incremented position having a second labeled operation, the first and second labeled operations each referring to an operation in removing the sterilization device from the elongated catheter; and wherein moving the release mechanism from the first incremented position to the second incremented position causes: activating the outer expandable portion of the sterilization device to expand inside the fallopian tube; and withdrawing the corewire from the expanded sterilization device to fully disengage the expanded sterilization device from the elongated catheter while keeping the endoscope and elongated catheter in a stable position rotationally relative to the patient $[33,34]$.

A composition comprising an antacid, and a local, topical anesthetic is used to relieve pain or discomfort associated with a sore throat (viral or bacterial infection, or a common or seasonal allergy), and therefore, the invention is also directed to a method of alleviating the pain or discomfort associated with a sore throat comprising instructing a human to orally administer the composition. The composition provides a controlled antacid delivery form for the administration of an antacid [35].

A method of topically applying an anesthetic or other medication, to one or both lips of the mouth is discussed by Jones. An applicator device is provided that has a curved first wall, a curved second wall, and a horizontal shelf that joins the first wall to the second wall. The first wall, the second wall and the horizontal shelf combine to define either one or two curved lip compartments. A volume of the anesthetic, or other medication, is placed within the curved lip compartments. The applicator device is partially placed in a person's mouth so that the second wall is positioned between the back of the lips and in front of the teeth. In this position, the lips of the user passes into the curved lip compartment and come in contacted by the anesthetic $[36,37]$.

\section{CONFLICT OF INTEREST}

The authors confirm that this article content has no conflict of interest.

\section{ACKNOWLEDGEMENTS}

We would like to thank Ms. Sonia Tofani, Mr. Carlo Drago for their precious help.

\section{REFERENCES}

[1] Rossi F, Cuomo V, Riccardi C. Pharmacology. Basic Principles and Therapeutic Applications. Minerva Medica: Chicago 2005.

[2] Mahmoud K, Ammar A. Immunomodulatory effects of anesthetics during thoracic surgery. Anesthesiol Res Prac 2011; 2011: 317410.

[3] Blum F, Zuo Z. Volatile anesthetics-induced neuroinflammatory and anti-inflammatory responses. Medical Gas Res 2013; 3:16.

[4] Fowler CJ. Possible involvement of the endocannabinoid system in the actions of three clinically used drugs. Trends Pharmacol Sci 2004; 25 (2): 59-61.

[5] Matta JA, Cornett PM, Miyares RL, Abe K, Sahibzada N, Ahern GP. General anesthetics activate a nociceptive ion channel to enhance pain and inflammation. Proc Natl Acad Sci USA 2008; 105(25): 8784-9.

[6] Morgan C, Curran HV. Ketamine use: A review. Addiction 2012; 107(1): 27-38.

[7] Vlisides P, Xie Z. Neurotoxicity of general anesthetics: An update. Curr Pharm Des 2012; 18(38): 6232-40.

[8] Berde CB, Strichartz GR. Local anesthetics. In: Miller RD, Eriksson LI, et al. Eds. Miller's Anesthesia. 7th ed. Philadelphia, Pa: Elsevier, Churchill Livingstone; 2009.

[9] Stiles P. Prielipp, Intralipid. Anesth Pa Saf Found Newsl 2009; 24(1). Retrieved June 2013.

[10] Benz EJ. Disorders of hemoglobin. In: Longo DL, Kasper DL, et al. eds. Harrison's Principles of Internal Medicine. $18^{\text {th }} \mathrm{Ed}$. New York, NY: McGraw Hill; 2012.

[11] Schatz M. Adverse reactions to local anesthetics. Immunol Allergy Clin North Am 1992; 12: 585-609.

[12] Nouette-Gaulain K. Local anesthetic in situ toxicity during peripheral nerve blocks: Update on mechanisms and prevention. Curr Opin Anaesthesiol 2012; 25(5): 589-95.

[13] Gillet A, Compère P, Lecomte F, Hubert P, Ducat E, Evrard B, et al. Liposome surface charge influence on skin penetration behavior. Int J Pharm 2011; 411:223-31.

[14] Kundu S, Achar S. Principles of office anesthesia: Part II. Topical anesthesia. Am Fam Physician 2002; 66: 99-102.

[15] Marra DE, Yip D, Fincher EF, Moy RL. Systemic toxicity from topically applied lidocaine in conjunction with fractional photothermolysis. Arch Dermatol 2006; 142: 1024-6.

[16] Package Insert EMLA. Astra USA, Inc. Westborough, Mass.

[17] Friedman PM, Mafong EA, Friedman ES, Geronemus RG. Topical anesthetics update: EMLA and beyond. Dermatol Surg 2001; 27: 1019-26.

[18] McKinlay JR, Hofmeister E, Ross EV, MacAllister W. EMLA cream-induced eye injury. Arch Dermatol 1999; 135(7): 855-6.

[19] Package inserts. ELA-Max. Ferndale Laboratories, Inc. Ferndale, Mich.

[20] Eichenfield LF, Funk A, Fallon-Friedlander S, Cunningham BB. A clinical study to evaluate the efficacy of ELA-Max (4\% liposomal lidocaine) as compared with eutectic mixture of local anesthetics cream for pain reduction of venipuncture in children. Pediatrics 2002; 109: 1093-9.

[21] Mehra P, Caiazzo A, Maloney P. Lidocaine toxicity. Anesth Prog 1998; 45: 38-41.

[22] Coudert AE, Ostertag A, Baaroun V, Artaud C, Ifi-Naulin C, Druo JP, et al. Phase III, randomized, double-blind, placebo-controlled trial of topical $2 \%$ lidocaine for the prevention and treatment of oral mucosal pain in children. Clin Oral Investig 2014; 18(4): 1189-94. 
[23] Cada DJ, Levien T, Baker DE. Lidocaine/tetracaine patch. Hosp Pharm 2006; 41: 265-73.

[24] Non-prescriptive drug therapy. Facts and Comparison: St Louis 1999; 630-8.

[25] Local anesthetics and ophthalmic drugs. Facts and Comparison: St Louis 1995; 38 (601-7, 1056)

[26] Package inserts. Alcain. Alcon, Humacao, Puerto Rico.

[27] Liesegang TJ, Perniciaro C. Fingertip dermatitis in an ophthalmologist caused by proparacaine. Am J Ophthalmol 1999; 127: 240-1.

[28] Smith RB, Everett WG. Physiology and pharmacology of local anesthetic agents. Int Ophthalmol Clin 1973; 13: 35-60.

[29] Gleich, G.J., Hunt, L.W. Method of treating neutrophil-related diseases with topical anesthetics. US6861449 (2005).

[30] Zasler, N.D., Carpenter, J. Application of topical anesthetics for modulation of neurogenic tremor. US7695733 (2010).
[31] Baez, T. Apparatus and methods for providing a medicinal substance applicator. US20120265155 (2012).

[32] McBride, E.V. Transdermal anesthetic applicator having thermochromic Indication. US8349358 (2013).

[33] Swann, B., Stout, C. A., Chan, K., Cruzada, J., Tunstall, K.A. Minimally invasive surgical stabilization devices and methods. US8360064 (2013).

[34] Nguyen, M., Aguilar, R., Swann, B., Aldridge, E. J., Stout, C.A., Bailey, P.A. Minimally invasive surgical stabilization devices and methods. US868504 (2014).

[35] Jones, T.L. Composition and method of treating a sore throat. US8362036 (2013).

[36] Jones, T.L. Method of treating a sore throat. US8367703 (2013).

[37] Placik, O.J. Device and method for topically applying medication to the lips. US8372047 (2013). 